\title{
Turbulence features of jet flow field in mine stopes
}

\author{
Xiaodong Wang ${ }^{1}$, Xiaoyun Wang ${ }^{2 *}$, Lin Lan ${ }^{1}$, Yuanyuan $\mathrm{Pu}^{3}$ \\ ${ }^{1}$ Faculty of Land Resources Engineering, Kunming University of Science and Technology, \\ Kunming 650093, China \\ ${ }^{2}$ College of Civil Engineering, Tongji University, Shanghai 200092, China \\ ${ }^{3}$ Number One Middle School of Huaning, Yuxi 652899, China
}

Email: 2026074073@qq.com

\begin{abstract}
This paper aims to explore the turbulence features of jet flow field in mine stopes. For this purpose, a chamber model was established at the scale of $1 / 20$ based on the similarity criterion. Then, the author obtained the average maximum jet length, width and the corresponding position, as well as the turbulent diffusion coefficient and the corresponding diffusion angle. Focusing on the turbulence features of jet flow velocity field, the distribution of turbulence intensity, skewness, flatness and Reynolds stress of the flow velocity in the crosssection were also acquired in the research. The results show that the flow velocity in the jet flow region belongs to the main airflow area when the air inlet and outlet are on the same side of the chamber, resulting in a satisfactory ventilation situation; the peak turbulence intensity in the cross-section and the peak Reynolds stress were both located near the axis and had the same order of magnitude. The research findings provide valuable references for the differentiation between the mainstream area and recirculation area in mine stopes.
\end{abstract}

Keywords: Dimensionless Coefficient, Jet Width, Jet Length, Turbulence Intensity, Reynolds Stress.

\section{AIMS AND BACKGROUND}

The research on jet injection has a long history, owing to the various forms and scales of the phenomenon in industrial production and nature. With the emergence of hot-wire anemometer and particle tracking velocimetry, the air jet pattern and fluid mechanics have been further studied by scholars [1-2]. The previous research has shown that the jet greatly boosts the cross-section velocity in the turbulent area and widens the diffusion angle; For stope ventilation, the air velocity and turbulence intensity distribution depend on the momentum ratio of air jet and the induced air [3]. To displace the dirty wind and dust in mine chambers, some scholars have carried out theoretical and experimental research on the smoke and dust in the tunnel [4-6]. For instance, Y. M. Wang [7] explored the removal of blasting fume and smoke in mine chambers by deriving the formula of ventilation capacity and turbulent mass transfer coefficient according to the ventilation process equation and turbulent diffusion coefficient.

With the development of production technology, the mining scale and chamber space are constantly increasing, leading to diverse and complex air distributions. Against this backdrop, it is very meaningful to study the turbulence features of jet flow field in stopes. Therefore, these features were examined in this research to optimize the ventilation plan of mine chambers.

\section{TEST DEVICE AND NOMENCLATURE}

\subsection{Device}


Figure 1. Sketch map of the test model

Based on the similarity criterion, a chamber model was established at the scale of $1 / 20$ (Figure 1). The model is 400 $\mathrm{cm}$ in length, $100 \mathrm{~cm}$ in width and $26 \mathrm{~cm}$ in height. The crosssections of air inlet and outlet are both $0.2 \mathrm{~cm} \times 0.2 \mathrm{~cm}$ in size. In the test, the inlet flow velocity of the turbulent jet is $u_{j}=1$ $\mathrm{m} / \mathrm{s}$; the ambient gas velocity of reverse flow is $u_{a}=0.1 \mathrm{~m} / \mathrm{s}$; the ratio of inlet flow velocity to ambient flow velocity is $u_{j} / u_{a}=10$. 


\subsection{Nomenclature}

For the convenience of the research, the relevant parameters of flow velocity features of the turbulent jet were defined as follows [8]:

Table 1. Parameters of the flow velocity features

\begin{tabular}{|c|c|}
\hline$u_{j}$ & The inlet flow velocity of the turbulent jet $(\mathrm{m} / \mathrm{s})$ \\
\hline$u_{a}$ & The ambient flow velocity $(\mathrm{m} / \mathrm{s})$ \\
\hline$u_{j} / u_{a}$ & $\begin{array}{l}\text { The jet flow velocity ratio (i.e. the ratio of inlet flow } \\
\text { velocity to ambient flow velocity) }\end{array}$ \\
\hline$u$ & $\begin{array}{l}\text { The actual flow velocity of a certain point in jet flow } \\
\text { field }\end{array}$ \\
\hline$u_{c}$ & $\begin{array}{l}\text { The actual flow velocity of a certain point on the jet } \\
\text { axis }\end{array}$ \\
\hline$u_{e}$ & $\begin{array}{l}\text { The additional flow velocity of a certain point in the } \\
\text { jet flow field; } u_{e}=u+u_{a}\end{array}$ \\
\hline$u_{e m}$ & $\begin{array}{l}\text { The additional flow velocity on the jet axis; } u_{e m}=u_{c}+ \\
u_{a}\end{array}$ \\
\hline$b_{g}, r_{e}$ & $\begin{array}{l}\text { The half width of the feature jet, whose additional } \\
\text { flow velocity in the cross-section of jet flow equals } \\
\text { the radial coordinate of } u_{e m} / e\end{array}$ \\
\hline$r$ & $\begin{array}{l}\text { The radial distance with the jet axis as the circle } \\
\text { centre in the cross-section of jet flow }\end{array}$ \\
\hline$b_{0}, r_{0}$ & $\begin{array}{l}\text { The distance from the position of zero actual flow } \\
\text { velocity in the cross-section of jet flow to the axis } \\
\text { (namely the maximum jet radius) }\end{array}$ \\
\hline$x_{p}$ & The average maximum jet depth \\
\hline$y_{b}, x_{b}$ & $\begin{array}{l}\text { The average maximum jet width and its } \\
\text { corresponding axial coordinate }\end{array}$ \\
\hline
\end{tabular}

\section{BOUNDARY FEATURES OF JET FLOW IN THE TEST MODEL}

\subsection{Flow velocity distribution in the cross-section}

Taking the position of $y=0.1$ as the circle centre, the author measured the flow velocity distribution in the cross-section of jet flow at $0.5 \mathrm{~m}, 1.0 \mathrm{~m}, 2.0 \mathrm{~m}, 3.0 \mathrm{~m}$ and $3.8 \mathrm{~m}$ along the $x$ axis away from the air inlet, respectively (Figure 2 ).

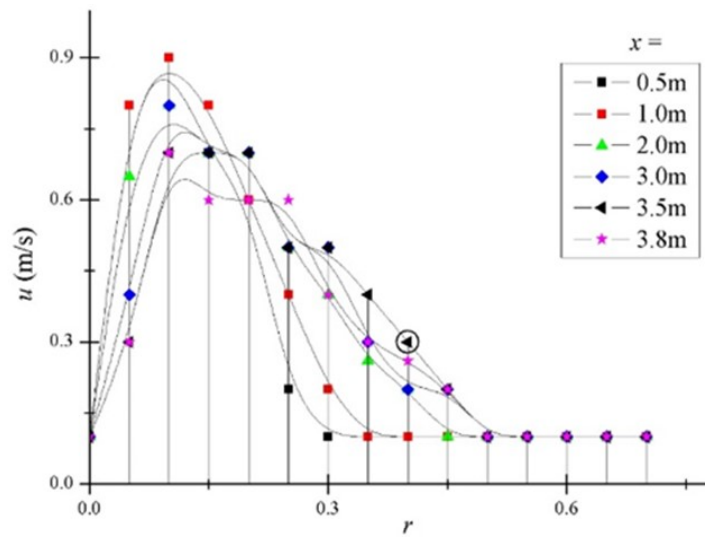

Figure 2. Flow velocity distribution in the cross-section of jet flow

In Figure 2, there are five speed curves in the cross-sections of jet flow along the $x$-axis. Before $x=1.0 \mathrm{~m}$ (the core area of jet flow), the shear layer of two cross-sections developed freely in the upstream, with a constant increase in the thickness of the layer. At $x=1.0 \mathrm{~m}$, the shear layers collided into each other violently, leading to self-similar distribution of various velocities in the cross-section. The flow velocity distribution curves in the cross-section of jet flow gradually flattened with the increase of $x$-axis coordinate, and the jet width increased along the jet. When $x<3.5 \mathrm{~m}$, the jet width in the cross-section increased constantly in the upstream; when $x>3.5 \mathrm{~m}$, the width plunged rapidly until it was consistent with the air outlet width of the chamber.

To disclose the effect of reverse flow velocity on the features of turbulent jet flow field, the dimensionless velocity $u_{e} / u_{e m}$ and the dimensionless width $r / r_{e}$ were taken as the vertical and horizontal coordinates, respectively. Then, the dimensionless additional flow velocity in the cross-section at different dimensionless distances was depicted in the same coordinate graph (Figure 3). Before the jet reached the maximum width, the dimensionless velocity exhibited obvious self-similarity in the cross-section of jet flow. As shown in Figure 3, the curve on the right side obeys the Gaussian distribution function:

$$
\frac{u_{e}}{u_{e m}}=e^{-\left(\frac{r}{r_{e}}\right)^{2}}
$$



Figure 3. Additional flow velocity distribution in the crosssection of jet flow

Compared with that in Figure 2, the additional flow velocity in Figure 3 needs a longer stream distance to reach the selfsimilar state. The speed curves are similar to each other at $x / D_{0}=5,10,15,20,25,30,35$ and 38 , and the corresponding distribution is known as the self-similar distribution. According to the measured results, the axial flow velocity gradually decreased with the increase in the $x$-axis coordinate. The dimensionless distribution relationship of additional flow velocity can be described with Gaussian distribution function, an evidence for the importance of downstream jet flow in the control of jet flow features.

\subsection{Expansion width of turbulent jet flow}

There existed a division surface between the jet flow in the reverse flow environment and the ambient gases in the chamber. On the surface, the average flow velocity approached zero. Figure 4 presents the variation in the dimensionless width $b_{0}$ between the zero flow velocity positions in the cross-section at different downstream and axial points of the jet. As shown in the figure, the jet flow in the chamber expanded linearly to the maximum width, and 
then dropped rapidly to the width of the outlet. The jet flow variation curve has a triangular shape. Figure 4 also illustrates the variation of half jet width $b_{g}$ with the additional flow velocity at different positions of the jet. The illustration indicates that the half width of the jet flow expanded linearly before the jet reached the maximum width.

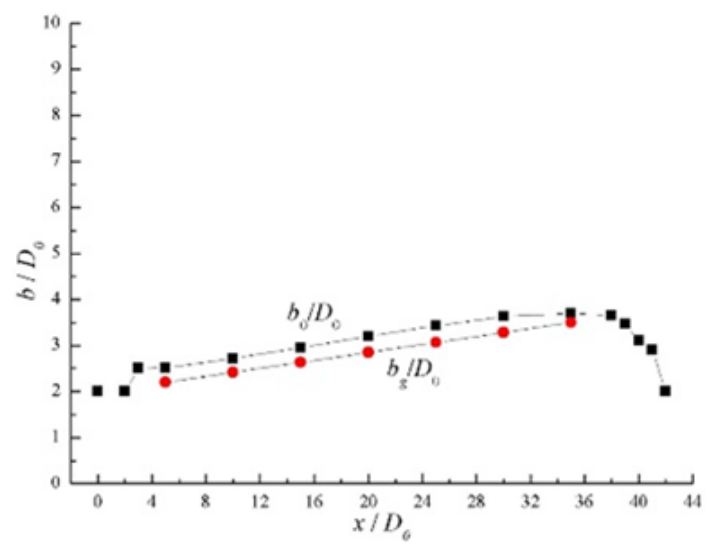

Figure 4. Variation of jet width along the jet

In light of the data results in Figure 4, the maximum jet depth $x_{b}$ and the average maximum jet width $y_{b}$ can be obtained as $y_{b} / D_{0}=3.7$ and $x_{b} / D_{0}=35$. In the ventilation practice, the jet flow is so turbulent that the boundary is an irregular surface interlaced with turbulent vortexes and ambient potential flows. In analysis, however, the jet boundary is generally viewed as a linearly extended surface.

Because the jet flow is the turbulent flow in the ventilation practice, the jet boundary actually is an irregular surface consisting of turbulent vortex and ambient potential flow interlacedly, but generally, in practical analysis the jet boundary is considered as the interface of linear extension. The diffusion angle, also known as the polar angle, is half of the maximum angle included in the boundary line outside the jet flow. The angle can be calculated as follows:

$\operatorname{tg} \beta=k \alpha$

where $\beta$ is the diffusion angle; $\mathrm{k}$ is test coefficient $(k=2.44$ for plane jet); $\alpha$ is the turbulence coefficient. The turbulence coefficient, the feature coefficient of jet structure, was determined by experiment.

In the experiment, the flow boundary along the jet was considered as a linearly extended structure. Then, the diffusion angle was calculated based on the average turbulence coefficient along the jet:

$\alpha=\frac{D_{0}}{X}\left(\frac{0.48 u_{j}}{u_{c}}-0.147\right)$

where $x$ is the jet distance $(\mathrm{cm}) ; D_{0}$ is the equivalent diameter of air outlet (m).

According to formulas (2) and (3), jet diffusion coefficient was derived as $\alpha=0.1628$, and corresponding diffusion angle was $13.19^{\circ}$. Next, the relationship between turbulence coefficient and dimensionless distance was obtained (Figure 5), using the turbulence coefficient $\alpha$ and the dimensionless distance $\mathrm{x} / \mathrm{D} 0$ as vertical and horizontal coordinates.

As can be seen from Figure 5, the turbulence coefficient of jet flow at the air inlet decreased with the increase in the axial distance $x$. The turbulence coefficient depicts the relationship between the axial distance $\mathrm{x}$ and dimensionless distance $x / D_{0}$. Nevertheless, the relationship in our experiment was relatively weak. In other words, the turbulence coefficient of jet flow varied little in the calculation interval, and can thus be considered as a constant.

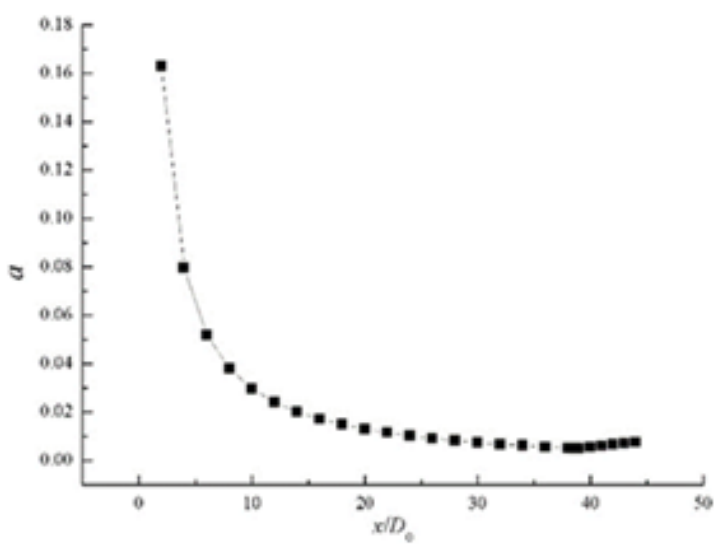

Figure 5. Relationship between turbulence coefficient and dimensionless distance

\subsection{Variation in flow velocity of jet axis along the jet}

With the dimensionless $u_{c} / u_{j}$ and $x / D_{0}$ as vertical and horizontal coordinates, the axial variation in the dimensionless flow velocity was calculated (Figure 6).

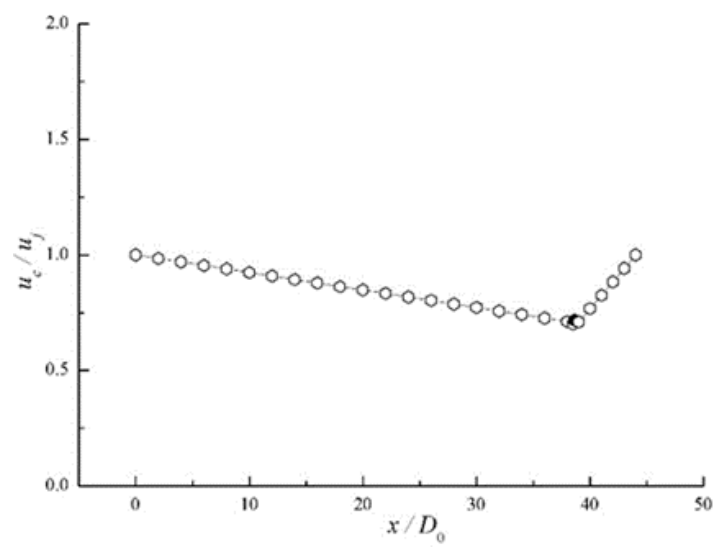

Figure 6. Axial variation in flow velocity

From Figure 6, it can be seen that the jet flow velocity underwent a sharp decrease once the jet flow entered into the stope. After dropping to $0.7 \mathrm{~m} / \mathrm{s}$, the flow velocity rebounded rapidly to the airflow velocity at the outlet. Then, the maximum jet depth under the test condition was obtained and expressed as $x_{p} / D_{0}=38.5$. Thus, the axial flow velocity can satisfy the ventilation requirements provided that the air inlet and air outlet are on the same side of the chamber.

Through the above tests, it is concluded that the airflow of the stope is a confined jet flow, with boundary parameters related to the relative length and width of the chamber. When the boundary width of jet flow was 0.37 times as much as the overall width of the chamber, both the jet width and flow velocity reached the maximum value. Therefore, the corresponding region is the main airflow area, and the most ventilated area in the test. During the development of jet flow, the total capacity of the main airflow area increased. However, the airflow continuity requires that the outflow capacity at the 
outlet must be equal to that at the inlet. Therefore, a part of the airflow started to flow reversely from the cross-section and collided with the developing jet flow, forming a circular flow with the jet flow boundary as the axis.

\section{TURBULENCE FEATURES OF JET FLOW VELOCITY FIELD}

\subsection{Turbulence intensity distribution in cross-section of jet flow}

Turbulence intensity can be expressed by the root mean square of fluctuating velocity as follows:

$$
u^{*}=\sqrt{\overline{u^{\prime 2}}}
$$

where $u^{*}$ is the longitudinal turbulence intensity; $u^{\prime}$ is the longitudinal fluctuating velocity.

Figure 7 shows the distribution of dimensionless turbulence intensity $u * / u_{c}$ of longitudinal flow velocity in the crosssection of jet flow, whose intensity is of the same order of magnitude.

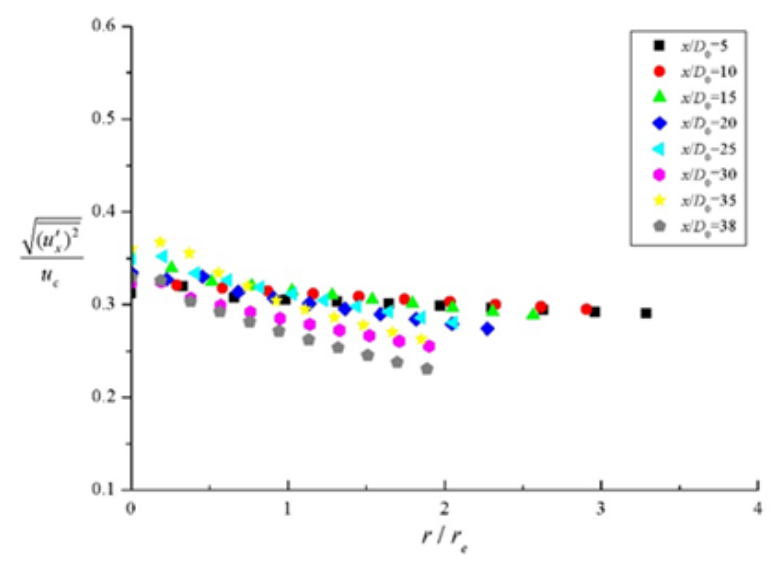

Figure 7. Turbulence intensity distribution in the crosssection

According to Figure 7, the turbulence intensity in the crosssection exhibited a certain self-similarity before the jet reached the maximum average width, and the peak turbulence intensity of flow velocity varied near 0.37 , which deviated from the axis of $r / r_{e}=0.15$. After the jet reached and surpassed the maximum average width, the dimensionless turbulence intensity distribution had no obvious regularity in the cross-section. Despite the gradual increase in the turbulence, the peak dimensionless maximum turbulence slowly moved towards the axis. Then, the axial flow velocity plunged, as the turbulent jet flow was diluted and mixed more fiercely due to the ambient gases of the reverse flow.

\subsection{Skewness and Flatness distribution in the jet flow cross-section}

As the function of third-order central moments [9], skewness was adopted to measure the deviation in the probability density distribution curve of flow velocity. Zero skewness means the curve shows normal distribution. Figure 8 shows the measured skewness of flow velocity in the crosssection of jet flow in our test.

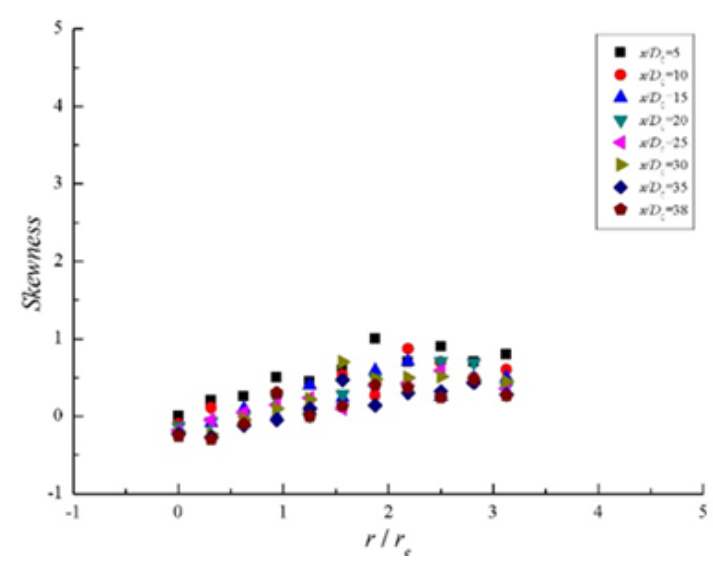

Figure 8. Distribution of flow velocity skewness in the crosssection

As shown in Figure 8, the skewness was zero near the central axis, and peaked at 1 in the position of $r / r_{e}=2$. After the skewness reached the cross-section of the average maximum width, the deviation coefficient showed no obvious regularity in distribution. This is because the jet energy dropped rapidly under the collision with the reverse flow once the jet reached the maximum average width.

Then, the flatness was introduced to measure the probability density distribution curve kurtosis of flow velocity, which is a function of four-order central moments. Figure 9 displays the measured flatness of flow velocity in the cross-section of jet flow in our test.



Figure 9. Distribution of flow velocity flatness in the crosssection

It can be seen from Figure 9 that the flatness fluctuated near zero with the dimensionless width at about $r / r_{e}=2$, and increased with the dimensionless width. Meanwhile, the collision with the reverse flow induced the rapid drop in jet energy of the chamber. Both measured skewness and flatness demonstrate the good self-similarity of flow velocity distribution in the cross-section.

4.3 Change relationship among dimensionless width, turbulence intensity, and Reynolds stress in the crosssection

The normal stress and the tangent stress of turbulent flow are collectively referred to as the Reynolds stress. The stress is the additional stress caused by the movement of turbulent 
fluctuations [10-11]. Figure 10 depicts the change relationship among dimensionless width, turbulence intensity, and Reynolds stress.

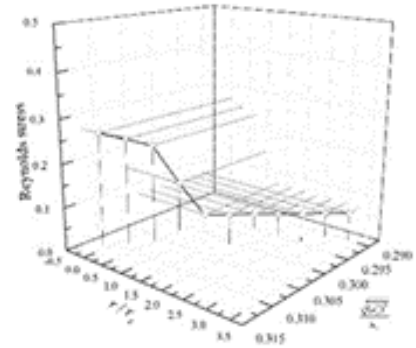

(a) $x / D_{0}=5$



(c) $x / D_{0}=15$

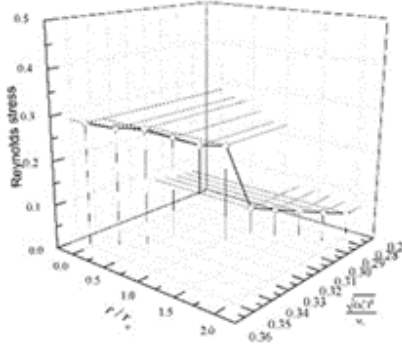

(e) $x / D_{0}=25$

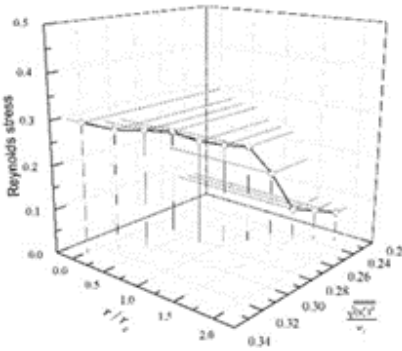

(g) $x / D_{0}=35$

(h) $x / D_{0}=38$

Figure 10. Change relationship among the dimensionless width, turbulence intensity, and Reynolds stress

According to Figure 10, the maximum Reynolds stress 0.295 occurred at $r / r_{e}=0$ in our test, with the $2 \mathrm{D}$ jet $\mathrm{n}$ the crosssection. The test data show that the Reynolds stress of jet flow had an obvious self-similarity after $x / D_{0}>10$ in the downstream. The Reynolds stress increased rapidly before $x /$ $D_{0}=10$ in the upstream, and then it drew closer to the same order of magnitude, with the peak value ranging from 0.279 to 0.295 within the self-similar region. The peak position tended to appear near the axis in the downstream at $x / D_{0}=10$, because the turbulent energy had a greater scope of influence along the jet edge and the axis after being transferred to the transverse component. When $x / D_{0}>10$, the downstream area was outside the core area of jet flow. In this case, the average velocity distribution could be illustrated by a "jet flow pattern" curve, which is different from the flat distribution. Therefore, the velocity gradient appeared in the chamber along the $y$ direction.

\section{CONCLUSIONS}

(1) This paper creates a chamber model to examine the boundary features of jet flow velocity field, and obtains the average maximum jet length, width and the corresponding position, as well as the turbulent diffusion coefficient and the corresponding diffusion angle.

(2) From the chamber inlet to the half width of the average maximum jet, the half jet width expanded linearly with a certain diffusion, and the axial flow velocity first decreased and then increased. Hence, the entire region of jet flow was the most ventilated area in the test.

(3) Focusing on the turbulence features of jet flow velocity field, the author also acquired the distribution of turbulence intensity, skewness, flatness and Reynolds stress of the flow velocity in the cross-section.

(4) It is discovered that the maximum turbulence intensity peaked in the axis, and the turbulence intensity generally decreased with the increase of dimensionless width and remained relatively uniform in the mainstream.

(5) As the dimensionless width widened, the skewness in the cross-section tended to zero and then exhibited an increasing trend. The flatness followed exact the same trend. The Reynolds stress of jet flow was self-similar in each crosssection, and its peak position lied close to the jet axis, with the same order of magnitude.

\section{ACKNOWLEDGMENT}

This research was supported by the China Postdoctoral Science Foundation project (2015M582763XB \& KKSY201721032).

\section{REFERENCES}

[1] Alfred K., Philip H., Tuve G.L. (1950). Comparative study of ventilating jets from various types of outlets, ASHVE Trans, Vol. 56, No. 1, pp. 459-478.

[2] Tuve G.L. (1953). Air velocities in ventilating jets, ASHVE Trans, Vol. 59, No. 1, pp. 261-282.

[3] Warda H.A., Kassab S.Z., Elshorbagy K.A., Elsaadawy E.A. (1999). An experimental investigation of the nearfield region of free turbulent round central and annular jets, Flow Meas Instrum, Vol. 10, No. 1, pp. 1-14. DOI: $\underline{\text { 10.1016/S0955-5986(98)00042-9 }}$

[4] Lin C.S., Moutton R.W., Puttnam G.L. (1953). Mass transfer between solid wall and fluid stream, mechanism and eddy distribution relationships in turbulent flow, Industrial \& Engineering Chemistry, Vol. 45, No. 1, pp. 636-640. DOI: 10.1021/ie50519a048

[5] Friendllander S.K., Johnstone H.F. (1957). Deposition of suspended particles from turbulent gas streams, Industrial \& Engineering Chemistry, Vol. 49, No. 7, pp. 1151-1156. DOI: $\underline{10.1021 / \mathrm{ie} 50571 \mathrm{a} 039}$ 
[6] Li E.L., Wang B.Q. (1989). The mathematical model of turbulent mass transfer in mines and longitudinal dispersion coefficient, J. NEU, Vol. 8, No. 3, pp. 65-69.

[7] Wang Y.M., Luan C.C. (1983). The calculation of air quantity required for flat-chamber stope, Chin Min Eng, Vol. 37, No. 1, pp. 26-32.

[8] Hua M., Wang H.M., Tang H.W. (2003). Effects of velocity ratios on characteristics of jets in counterflowing stream, SH Environ Sci, Vol. 22, No. 5, pp. 313316.

[9] Hua M., Tang H.W., Wang H.M. (2000). Experiment of turbulence characteristics in round jet flow field measured with ADV, Hydro-Sci Eng, Vol. 3, No, 4, pp. 18-21.

[10] Li W.P., Wang X.Q., Xie H. (2007). Phase delay of the Reynolds stress and wall pressure fluctuation in wall shear flows, Journal of Huazhong University of Science \& Technology, Vol. 35, No. 4, pp. 122-124.

[11] Zhang Y., Liu S.Y., Wang B.G. (2005). Application of the Reynolds stress model to the calculation of threedimensional turbulent flow-field, Journal of Aerospace Power, Vol. 20, No. 4, pp. 573-576. 